\title{
Echinococcal disease of the bone: An unusual cause of a pathological fracture
}

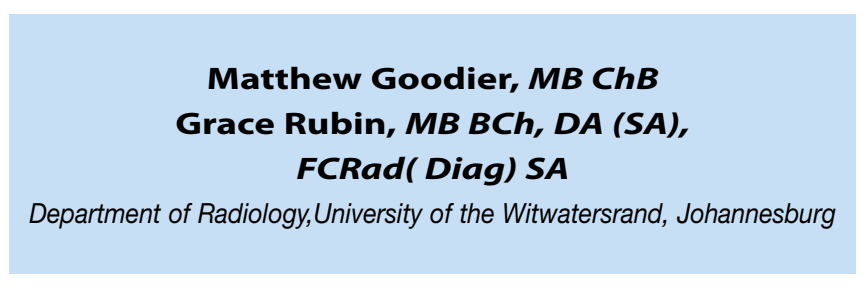

\section{Abstract}

Echinococcosis is caused by the larva of the tapeworm, Echinococcus granulosus or Echinococcus multiloccularis and is endemic in many rural areas of southern Africa. Echinococcosis of the bone is an unusual

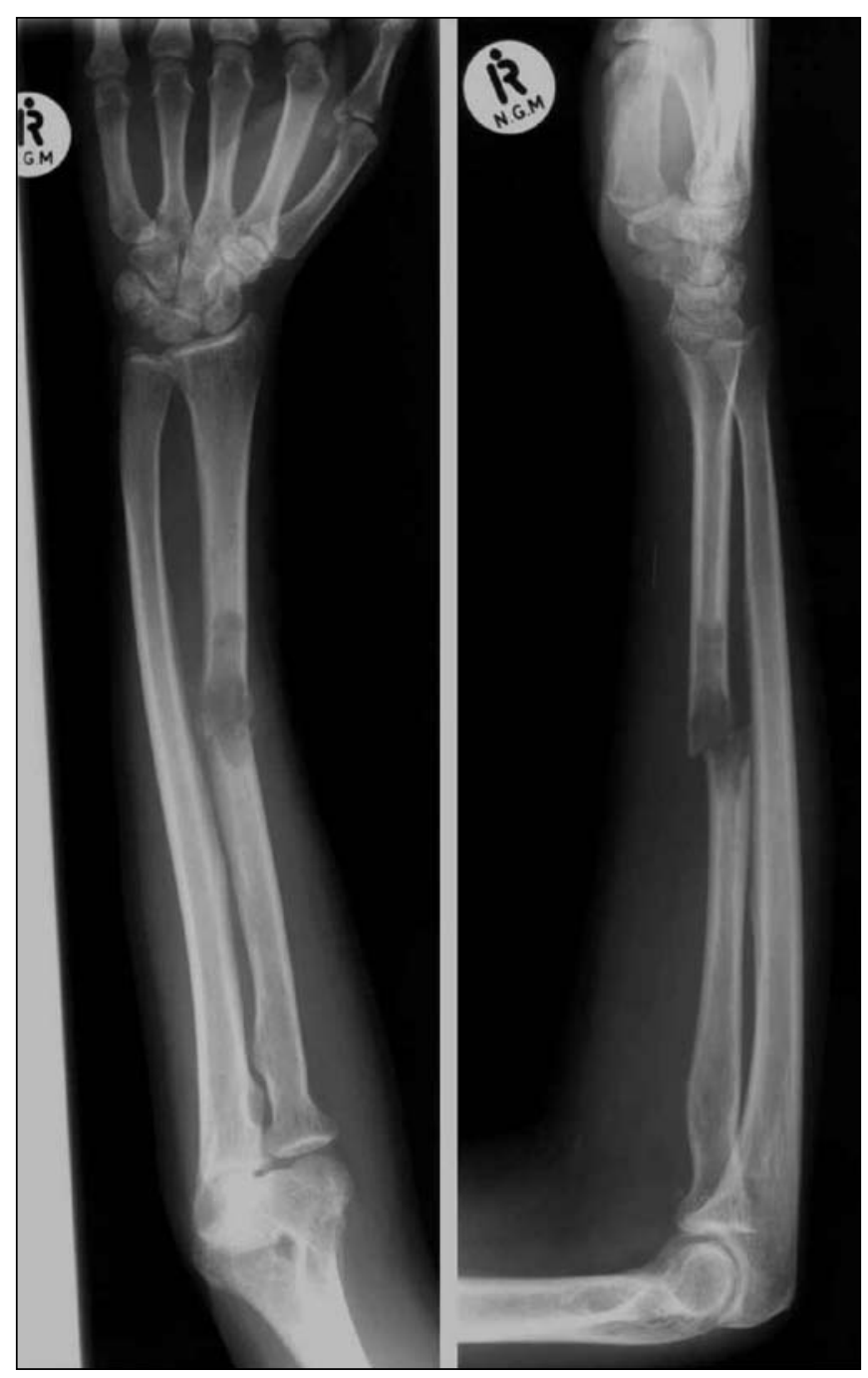

Fig. 1. Plain radiographs demonstrate a multilocular lucent lesion in the midshaft of the right radius, complicated by pathological fracture.

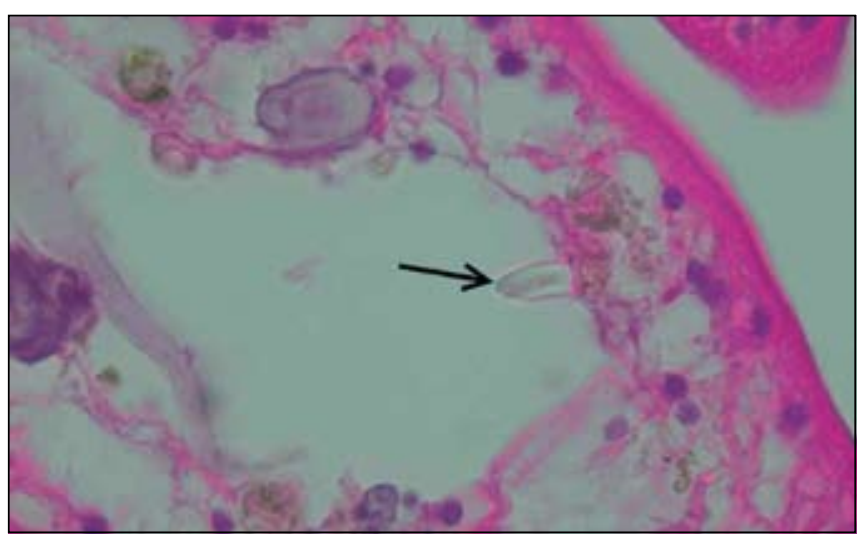

Fig. 2. Histological slide indicating the presence of a hydatid hooklet (arrow) (Figure by courtesy of the Division of Anatomical Pathology/NHLS, University of the Witwatersrand).

manifestation of echinococcal disease and a rare cause of a lytic lesion of bone. This report describes a 30-year-old woman who presented with an echinococcal cyst of the right radius complicated by a pathological fracture.

\section{Case presentation}

A 30-year-old woman presented to the casualty department of Helen Joseph Hospital complaining of right forearm pain after a minor trauma. Although currently residing in Johannesburg, she had spent most of her life on a farm near Polokwane.

Anteroposterior (AP) and lateral radiographs of the right radius and ulna (Fig. 1) revealed a pathological transverse fracture of the mid-shaft of the radius. There was an underlying multiloculated lytic lesion with a narrow zone of transition. There was endosteal scalloping with thinning of the cortex but no bony expansion. There was no associated periosteal reaction or soft-tissue mass.

The patient was taken to theatre where the lesion was curetted, the defect filled with bone chips from an iliac autograft, and internal fixation performed. At operation, partial cortical erosion was noted and the cyst was found to contain gritty, gelatinous material with a thick white membrane.

The microbiology report indicated the presence of hydatid hooklets. This was confirmed on histology which demonstrated the presence of the laminated and germinative layers of a hydatid cyst (Fig. 2). The indirect haemagglutination assay (IHA) for hydatid disease was negative. Chest X-ray, ultrasound of the abdomen and radiographs of the other long bones were normal. Postoperatively, the patient was placed on albendazole $400 \mathrm{mg}$ bd.

\section{Discussion}

Echinococcosis is caused by the larva of the tapeworm Echinococcus granulosus or Echinococcus multiloccularis and is endemic in many 


\section{CASE REPORT}

rural areas of southern Africa. The disease is acquired when man becomes the intermediate host of the parasite by ingestion of ova either through contact with dogs (the definitive host) or environmental contamination. ${ }^{1}$

Echinococcal cysts usually involve the liver or lung; involvement of the bone is rare, accounting for $0.5-4 \%$ of all cases of echinococcosis. ${ }^{1}$ When the bone is involved, the most common site is the spine, followed by the pelvis, femur, tibia, ribs, skull, scapula, humerus and fibula. ${ }^{2}$ Involvement of the radius, as in our case, appears to be especially rare, although isolated cases have been reported. ${ }^{3}$

The disease is usually clinically silent until a complication occurs. In lesions of the long bones, pathological fracture is the most common presentation. ${ }^{2}$ Uncommonly, the cyst may become secondarily infected, resulting in osteomyelitis with formation of an abscess and draining sinus to the overlying skin.

The diagnosis is usually suggested by a combination of radiological and serological findings, and then confirmed intraoperatively. As expected, the IHA serological test in our patient was negative. This is because cysts in the bone seldom induce detectable levels of host antibody.

Radiographs usually show a lytic lesion which may be unilocular or more commonly multilocular. This is because, in echinococcal cysts of the bone, the pericyst layer, which usually forms a connective tissue barrier to spread of the disease, does not form. ${ }^{1}$ The margins may be wellor ill-defined. The most common site is the metaphysis. Involvement of the epiphysis and articular surface of the bone is uncommon. Periosteal reaction usually indicates the presence of a pathological fracture. If the disease extends through the cortex, an associated soft-tissue mass may be seen. If the cyst remains intraosseous, internal calcifications are uncommon. ${ }^{2}$

Abdominal ultrasonography and chest radiography are routinely performed to detect liver and lung cysts, which may co-exist with bone cysts in up to $45 \%$ of patients. ${ }^{5}$ Both MRI and CT help to define the exact extent of the lesion, with CT defining internal calcifications and MRI allowing better depiction of any extra-osseous soft-tissue extension and nerve involvement.

Differential diagnosis includes expansile lytic lesions of bone, such as aneurysmal bone cyst, simple bone cyst, enchondroma and fibrous dysplasia, as well as giant cell tumour if the lesion is situated in the epiphysis.

Surgery is the treatment of choice. Because of the high recurrence rate following curettage, radical resection of the involved segment with bone grafting is preferred. ${ }^{6}$ If the diagnosis is suspected preoperatively, adjuvant medical therapy with imidazole derivatives (e.g. albendazole) may be initiated preoperatively and continued postoperatively to control systemic spread and prevent local recurrence.

\section{Conclusion}

Echinococcal cyst of bone is a rare manifestation of echinococcal disease, with involvement of long bones, such as the radius, especially rare. Nevertheless, the diagnosis should always be considered in the radiological differential of a lytic lesion of the bone, especially in a patient who has spent time in an endemic area. Making the diagnosis preoperatively by means of radiological and serological methods is important as this allows initiation of preoperative imidazole therapy as well as suggesting the most appropriate form of surgical management.

1. Pedrosa I, Saíz A, Arrazola J, Ferreirós J Pedrosa CS. Echinococcosis disease: Radiographic and pathologic features and complications. RadioGraphics 2000;20:795-817.

2. Polat P, Kantarci M, Alper F, Suma S, Koruyucu MB, Okur A. Echinococcosis disease from head to toe. RadioGraphics 2003; 23:475-494.

3. Ammari F, Khasawneh Z, Salem M, Mesmar M, Omari H, Masádeh R. Hydatid disease of the musculoskeletal system. Surgery 1998;124:934-937.

4. Eckhert J, Deplazes P. Biological, epidemiological, and clinical aspects of echinococcosis, a zoonosis of increasing concern. Clin Microbiol Rev 2004;17:107-135.

5. Herrera A, Martinez AA, Rodriguez J. Spinal hydatidosis. Spine 2005;30:2439-2444.

6. Sayek I, Tirnaksiz MB, Dogan R. Cystic echinococcosis disease: current trends in diagnosis and management. Surg Today 2004;34:987-996. 